\title{
Si-DOPED InAs/GaAs QUANTUM DOT SOLAR CELL WITH AIAs CAP LAYERS
}

\author{
Dongyoung Kim ${ }^{(1)}$, *, Mingchu Tang ${ }^{(1)}$, Jiang Wu ${ }^{(1)}$, Sabina Hatch (1), Yurii Maidaniuk ${ }^{(2)}$, Vitaliy Dorogan ${ }^{(2)}$, \\ Yuriy I. Mazur ${ }^{(2)}$, Gregory J. Salamo ${ }^{(2)}$, Huiyun Liu ${ }^{(1)}$ \\ (1) Department of Electronic and Electrical Engineering, University College London, Torrington Place, \\ London WC1E 7JE, United Kingdom, \\ (2) Institute for Nanoscience and Engineering, University of Arkansas, Fayetteville, \\ Arkansas 72701, United States of America \\ *Email:d.kim@ucl.ac.uk
}

\begin{abstract}
In this work, the effect of $\mathrm{Si}$ doping on InAs/GaAs quantum dot solar cells with AlAs cap layers is studied. The AlAs cap layers suppress the formation of the wetting layer during quantum dot growth. This helps achieve quantum dot state filling, which is one of the requirements for strong sub-bandgap photon absorption in the quantum dot intermediate band solar cell, at lower $\mathrm{Si}$ doping density. Furthermore, the passivation of defect states in the quantum dots with moderate $\mathrm{Si}$ doping is demonstrated, which leads to an enhancement of the carrier lifetime in the quantum dots, and hence the open-circuit voltage.
\end{abstract}

\section{INTRODUCTION}

Since the concept of the intermediate band solar cell (IBSC) was proposed in 1997, significant efforts have been made to realise IBSCs with efficiencies that exceed the Shockley-Queisser limit of $31 \%$ [1]. The IBSC utilises a collection of intermediate levels within the bandgap, called the intermediate band (IB), to absorb sub-bandgap energy photons. Its potential to exceed the Shockley-Queisser limit and reach the theoretical limit of $63.2 \%$ [2] arises from the additional photocurrent generated by the sub-bandgap photon absorption. One of the candidates for the high-efficiency IBSC is the quantum dot intermediate band solar cell (QD-IBSC), which utilises the discrete nature of the carrier density of states and the bandgap tunability of quantum dots (QDs). In a QD-IBSC, the IB is formed by the confined electron states in the QD array.

However, there have been several challenges in implementing high-efficiency QD-IBSCs. Firstly, thermal decoupling needs to be achieved between the IB and the conduction band (CB) of wetting layer (WL). The thermal coupling between the IB and $\mathrm{CB}$ enables the carriers to be thermally excited or relaxed between the IB and the CB [3]-[5]. This thermal process leads to suppression of the second-photon absorption, and hence a lower open-circuit voltage $\left(\mathrm{V}_{\mathrm{OC}}\right)$ [6], [7]. Tutu et al. have demonstrated suppression of thermal escape of electrons in QDs by removing the WL [8]. Lam et al. have also reported reduction of thermal coupling of QD states from the WL by introducing a potential barrier between the QDs and WL via Si doping [9]. Secondly, the accumulated strain from the QDs leads to the formation of threading dislocations [10]-[12]. The strain-induced dislocations of the QDs result in short minority carrier lifetime, and cause difficulties in increasing the number of the QD layers that are needed to maximise the photon absorption by QDs [11]-[14]. Furthermore, the performance of IBSCs relies on an IB that is partially filled with electrons [15], [16]. For strong sub-bandgap photon absorption, the IB needs to have empty states to receive the electrons pumped from the VB, and states filled with electrons to pump electrons to the CB. Luque et al. proposed the use of doping as a method to achieve partial filling of the confined states in the IB [17].

In this work, we report the saturation of strain-induced dislocations and QD state filling by Si-doping QDs in InAs/GaAs QDSCs with AlAs CLs. Previously, we demonstrated that the deposition of AlAs CLs on InAs QDs could suppress the formation of the WL [8]. Consequently, the effective bandgap of the QDSC was increased, which in turn led to the increase in the thermal activation energy and the $\mathrm{V}_{\mathrm{OC}}$. In this study, in addition to the enhancement of the $\mathrm{V}_{\mathrm{OC}}$ by applying AlAs CLs, a further increase in the $\mathrm{V}_{\mathrm{OC}}$ by $\sim 44 \mathrm{mV}$ is observed by passivating the defect states with moderate Si doping (6 e/dot). In addition, QD state filling, which is essential for strong two-photon absorption, is observed with significantly lower Si doping densities when compared with that of our previous study [9].

\section{GROWTH DETAILS}

The InAs/GaAs QDSC samples with AlAs CLs were grown by a solid-source molecular beam epitaxy (MBE) on $\mathrm{n}^{+}$GaAs (100) substrates. All SCs were grown with the same p-i-n structure that consists of a $200 \mathrm{~nm} \mathrm{n}^{+}$ GaAs buffer layer, $30 \mathrm{~nm} \mathrm{n}^{+} \mathrm{Al}_{0.35} \mathrm{Ga}_{0.65} \mathrm{As}$ back surface field, $1000 \mathrm{~nm} \mathrm{n}^{-}$GaAs base, $250 \mathrm{~nm} \mathrm{p} \mathrm{GaAs} \mathrm{emitter,}$ $30 \mathrm{~nm} \mathrm{p} \mathrm{Al}_{0.75} \mathrm{Ga}_{0.25} \mathrm{As}$ window layer, and $\mathrm{p}^{+} 50 \mathrm{~nm}$ GaAs contact layer. The intrinsic region of the SCs consists of 20 stacks of InAs QDs with 2 ML AlAs CLs 
separated by a $20 \mathrm{~nm}$ GaAs spacer. The QDs were grown by the Stranski-Krastanov mode at $\sim 500{ }^{\circ} \mathrm{C}$. The QDSCs were Si-doped with four different doping densities of $0,6,12$, and 18 e/dot.

Atomic-force microscopy (AFM) was used to analyse the morphology of the InAs QDs grown on GaAs. The average diameter of the QDs measured from the AFM image (shown in Figure 1) was $\sim 30 \mathrm{~nm}$, with an average height of $\sim 5 \mathrm{~nm}$. No large defective clusters were observed, which indicates a high structural quality [18]. The in-plane density of the InAs QDs was estimated to be $\sim 2.3 \times 10^{10} \mathrm{~cm}^{-2}$.

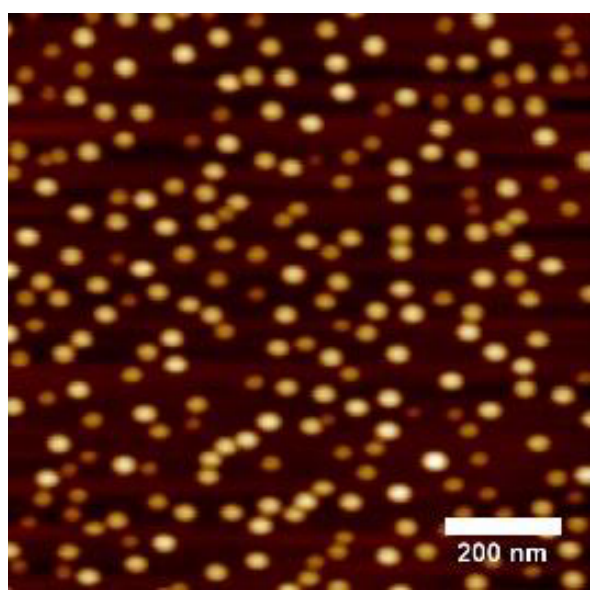

Figure 1 AFM images of InAs QDs grown on GaAs in $2 D$ (top) and $3 D$ (bottom).
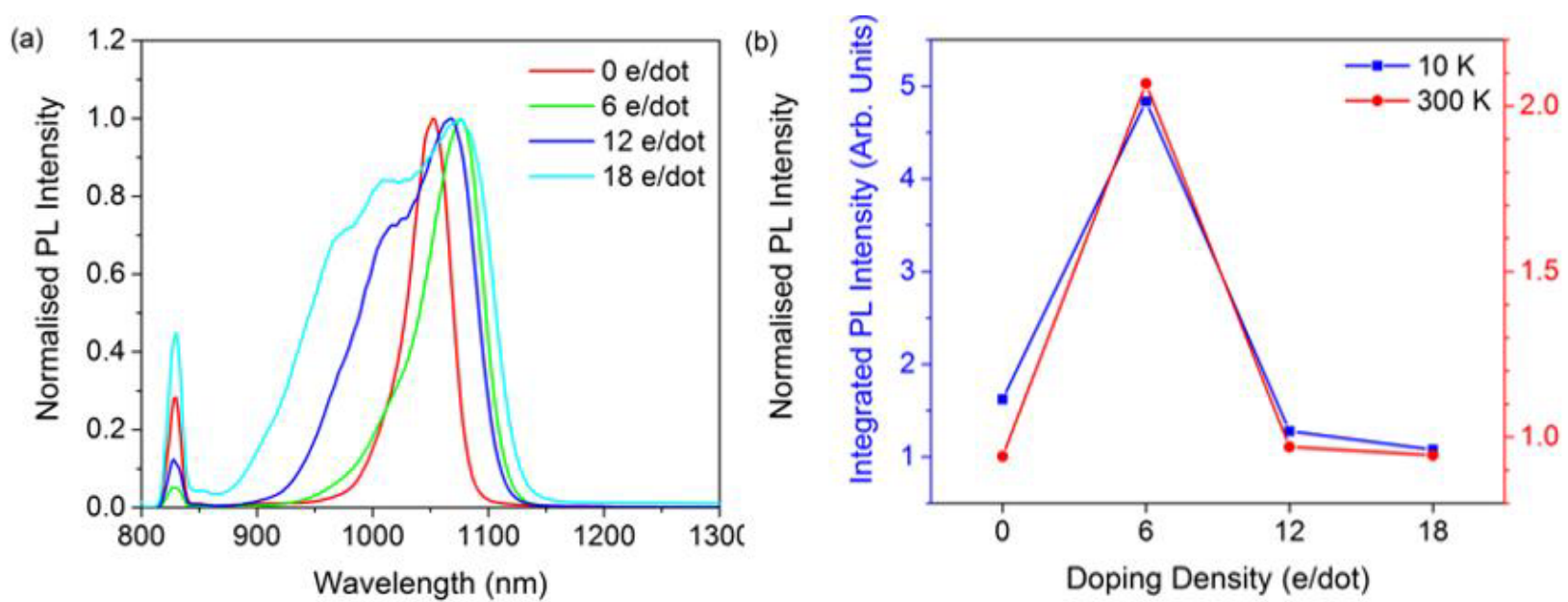

Figure 2 (a) Normalised PL spectra of Si-doped QDSCs with AlAs cap layers measured at $10 \mathrm{~K}\left(P_{\text {ex }}=37 \mathrm{~mW}\right)$, and (b) Integrated PL intensity vs. Si doping density at $10 \mathrm{~K}\left(I_{e x}=386 \mathrm{~W} / \mathrm{cm}^{2}, \lambda_{e x}=532 \mathrm{~nm}\right)$ and $300 \mathrm{~K}\left(I_{e x}=459\right.$ $W / \mathrm{cm}^{2}, \lambda_{\text {ex }}=635 \mathrm{~nm}$ ). 


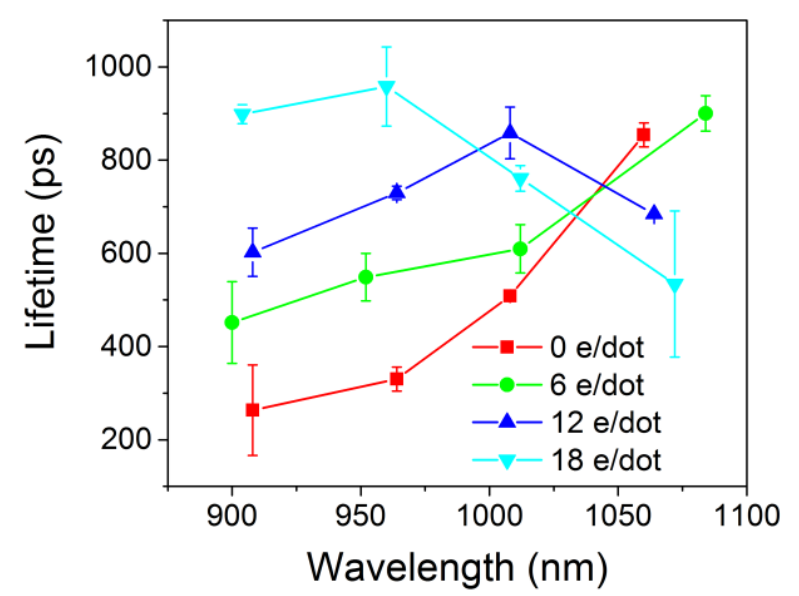

Figure 3 Carrier lifetime versus wavelength obtained from the transient photoluminescence spectra of the Sidoped QDSCs with AlAs cap layers at $10 \mathrm{~K}\left(\lambda_{e x}=750\right.$ nm)

In order to provide further evidence of the QD state filling and the passivation of defect states achieved by Si doping, a time-resolved PL study was performed for the QDCSs at $10 \mathrm{~K}$. Figure 3 shows that the carrier lifetime in the region of $875-925 \mathrm{~nm}$ increases with increasing Si doping density. This can be linked to the state filling effect observed from the steady-state PL measurements in Figure 2 (a). The appearance of the higher-energy QD emission peaks with higher Si doping densities in Figure 2 (a) indicates that the additional electrons supplied by the Si dopants are used to fill the QD states. With more QD states filled with electrons, fewer available decay channels exist for the carriers, which in turn results in longer carrier lifetimes. In contrast, the carrier lifetime of the QD ground state $(1050-1100 \mathrm{~nm})$ in Figure 3 shows that increasing the Si doping density leads to an initial increase with moderate doping density (6 e/dot), followed by a significant drop in the carrier lifetime with higher doping densities $(12,18 \mathrm{e} / \mathrm{dot})$. This trend is similar to that observed in the integrated PL intensity measurements, as shown in Figure 2 (b). Therefore, it can be inferred that the initial increase in the carrier lifetime with doping density of 6 e/dot originates from the passivated defect states. However, once all defect states are filled, further addition of Si atoms leads to the formation of non-radiative recombination centres, and/or increase Auger scattering rate, which decreases the carrier lifetime.

\section{SOLAR CELL PERFORMANCES}

The EQE spectra of the Si-doped QDSCs with AlAs CLs are presented in Figure 4. For all doped samples, there is a drop in EQE at $\sim 870 \mathrm{~nm}$ which corresponds to the bandgap of GaAs. At the higher wavelengths, flat spectral responses without a WL peak $(\sim 915 \mathrm{~nm})$ are observed. It can be also noted from Figure 4 that the supra-bandgap (400 - $870 \mathrm{~nm})$ absorption of all QDSCs with $\mathrm{Si}$ doping is significantly reduced when compared with that of the undoped QDSC. This can be attributed to the decrease in depletion region after $\mathrm{Si}$ doping, which reduces the effective absorption area.

The J-V characteristics of the QDSCs in Figure 5 show a clear dependence on the Si-doping density. Firstly, all Si-doped samples display a significant drop in the current density when compared with the undoped sample. This can be related to the reduction in the EQE contribution from the supra-bandgap region observed in Figure 4. Secondly, a further gradual decrease in the current density with increasing doping is observed amongst the doped QDSCs. This is likely to be due to the point defects formed by Si dopants substituting $\mathrm{Ga}$ and As or existing as interstitials [22], [23]. Although Si doping has a negative impact in the current density, an enhancement of the $V_{O C}$ is observed with moderate doping density, which is likely to be due to the moderate Si doping passivating the defect states.

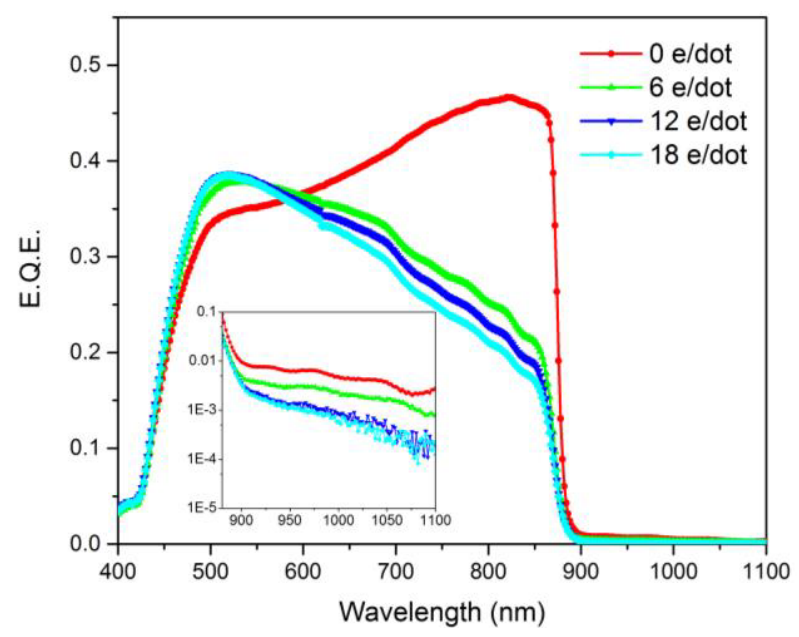

Figure 4 Linear-scale external quantum efficiency spectra of Si-doped QDSCs with AlAs cap layers. Inset shows semi-log scale of sub-bandgap external quantum efficiency. 


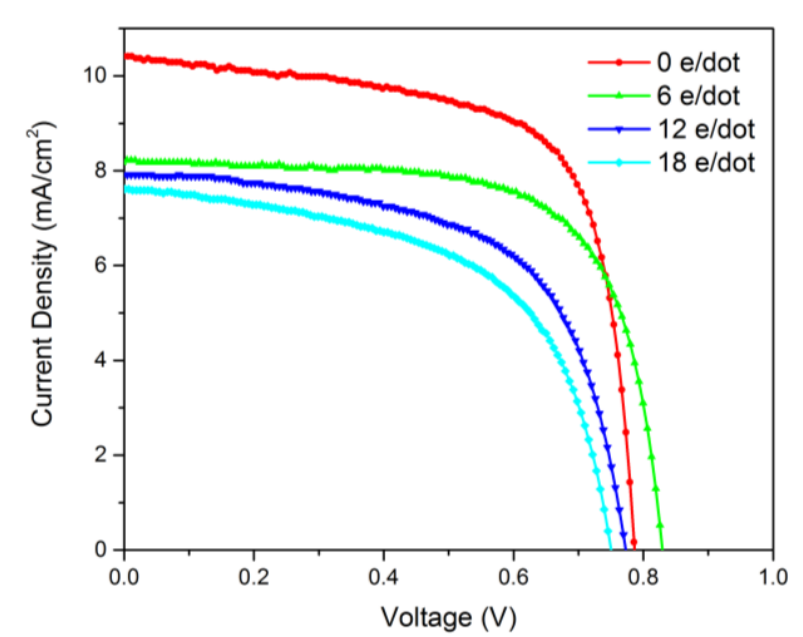

Figure 5 Current density versus voltage behaviour of Sidoped QDSCs with AlAs cap layers under one-sun (AM $.5 G)$ illumination

\section{CONCLUSION}

In conclusion, an improvement in the $\mathrm{V}_{\mathrm{OC}}(\sim 44 \mathrm{mV})$ has been achieved by introducing direct $\mathrm{Si}$ doping to QDs with AlAs CLs. The increase in the $\mathrm{V}_{\mathrm{OC}}$ is attributed to the passivation of the defect states with moderate Si doping (6 e/dot), alongside the reduced CBIB thermal coupling with AlAs CLs. Also, with the AlAs CLs, the QD state filling effect is observed with low Si doping densities. The factors that contributed to the decrease in the supra-bandgap EQE and the photocurrent after Si doping, such as the decrease in depletion region and the formation of point defects, should be taken into account when designing future Sidoped QDSCs. Nonetheless, the results presented in this article hold some promise for overcoming some of the main challenges in implementing QD-IBSCs with the efficiencies close to that of the theoretical model of the IBSC.

\section{REFERENCES}

[1] W. Shockley and H. J. Queisser, 'Detailed Balance Limit of Efficiency of p-n Junction Solar Cells', $J$. Appl. Phys., vol. 32, no. 3, pp. 510-519, Mar. 1961.

[2] A. Luque, A. Martí, and C. Stanley, 'Understanding intermediate-band solar cells', Nat. Photonics, vol. 6, no. 3, pp. 146-152, Mar. 2012.

[3] S. Sanguinetti, M. Henini, M. Grassi Alessi, M. Capizzi, P. Frigeri, and S. Franchi, 'Carrier thermal escape and retrapping in self-assembled quantum dots', Phys. Rev. B, vol. 60, no. 11, pp. 8276-8283, Sep. 1999.

[4] E. Antolín, A. Martí, C. D. Farmer, P. G. Linares, E. Hernández, A. M. Sánchez, T. Ben, S. I. Molina, C. R. Stanley, and A. Luque, 'Reducing carrier escape in the InAs/GaAs quantum dot intermediate band solar cell', J. Appl. Phys., vol. 108, no. 6, p. 64513, Sep. 2010.

[5] A. Luque and A. Martí, 'The Intermediate Band Solar Cell: Progress Toward the Realization of an Attractive Concept', Adv. Mater., vol. 22, no. 2, pp. 160-174, Nov. 2010.

[6] C. G. Bailey, D. V. Forbes, R. P. Raffaelle, and S. M. Hubbard, 'Near $1 \mathrm{~V}$ open circuit voltage InAs/GaAs quantum dot solar cells', Appl. Phys. Lett., vol. 98, no. 16, p. 163105, Apr. 2011.

[7] A. Mellor, A. Luque, I. Tobías, and A. Martí, 'Realistic Detailed Balance Study of the Quantum Efficiency of Quantum Dot Solar Cells', Adv. Funct. Mater., vol. 24, no. 3, pp. 339-345, Jan. 2014.

[8] F. K. Tutu, P. Lam, J. Wu, N. Miyashita, Y. Okada, K.-H. Lee, N. J. Ekins-Daukes, J. Wilson, and H. Liu, 'InAs/GaAs quantum dot solar cell with an AlAs cap layer', Appl. Phys. Lett., vol. 102, no. 16, p. 163907, Apr. 2013.

[9] P. Lam, S. Hatch, J. Wu, M. Tang, V. G. Dorogan, Y. I. Mazur, G. J. Salamo, I. Ramiro, A. Seeds, and H. Liu, 'Voltage recovery in charged InAs/GaAs quantum dot solar cells', Nano Energy, vol. 6, pp. 159-166, May 2014.

[10] H. Y. Liu, I. R. Sellers, T. J. Badcock, D. J. Mowbray, M. S. Skolnick, K. M. Groom, M. Gutiérrez, M. Hopkinson, J. S. Ng, J. P. R. David, and R. Beanland, 'Improved performance of $1.3 \mu \mathrm{m}$ multilayer InAs quantum-dot lasers using a highgrowth-temperature GaAs spacer layer', Appl. Phys. Lett., vol. 85, no. 5, pp. 704-706, Aug. 2004.

[11] F. K. Tutu, I. R. Sellers, M. G. Peinado, C. E. Pastore, S. M. Willis, A. R. Watt, T. Wang, and H. Y. Liu, 'Improved performance of multilayer InAs/GaAs quantum-dot solar cells using a highgrowth-temperature GaAs spacer layer', J. Appl. Phys., vol. 111, no. 4, p. 46101, Feb. 2012.

[12] A. Martí, N. López, E. Antolín, E. Cánovas, A. Luque, C. R. Stanley, C. D. Farmer, and P. Díaz, 
'Emitter degradation in quantum dot intermediate band solar cells', Appl. Phys. Lett., vol. 90, no. 23, p. 233510, Jun. 2007.

[13] X. Yang, K. Wang, Y. Gu, H. Ni, X. Wang, T. Yang, and Z. Wang, 'Improved efficiency of InAs/GaAs quantum dots solar cells by Si-doping', Sol. Energy Mater. Sol. Cells, vol. 113, pp. 144147, Jun. 2013.

[14] T. Sugaya, Y. Kamikawa, S. Furue, T. Amano, M. Mori, and S. Niki, 'Multi-stacked quantum dot solar cells fabricated by intermittent deposition of InGaAs', Sol. Energy Mater. Sol. Cells, vol. 95, no. 1, pp. 163-166, Jan. 2011.

[15] A. Martí, L. Cuadra, and A. Luque, 'Partial filling of a quantum dot intermediate band for solar cells', IEEE Trans. Electron Devices, vol. 48, no. 10, pp. 2394-2399, Oct. 2001.

[16] A. Martí, N. López, E. Antolín, E. Cánovas, C. Stanley, C. Farmer, L. Cuadra, and A. Luque, 'Novel semiconductor solar cell structures: The quantum dot intermediate band solar cell', Thin Solid Films, vol. 511-512, pp. 638-644, Jul. 2006.

[17] A. Luque and A. Martí, 'A metallic intermediate band high efficiency solar cell', Prog. Photovolt. Res. Appl., vol. 9, no. 2, pp. 73-86, Mar. 2001.

[18] H. Y. Liu, I. R. Sellers, M. Gutiérrez, K. M. Groom, W. M. Soong, M. Hopkinson, J. P. R. David, R. Beanland, T. J. Badcock, D. J. Mowbray, and M. S. Skolnick, 'Influences of the spacer layer growth temperature on multilayer InAs/GaAs quantum dot structures', J. Appl. Phys., vol. 96, no. 4, pp. 1988-1992, Aug. 2004.

[19] M. V. Marquezini, M. J. S. P. Brasil, J. A. Brum, P. Poole, S. Charbonneau, and M. C. Tamargo, 'Study of temperature-dependent exciton dynamics in a single quantum well with self-assembled islands', Surf. Sci., vol. 361-362, pp. 810-813, Jul. 1996.

[20] K. A. Sablon, J. W. Little, V. Mitin, A. Sergeev, N. Vagidov, and K. Reinhardt, 'Strong Enhancement of Solar Cell Efficiency Due to Quantum Dots with Built-In Charge', Nano Lett., vol. 11, no. 6, pp. 2311-2317, 2011.

[21] T. Inoue, S. Kido, K. Sasayama, T. Kita, and O. Wada, 'Impurity doping in self-assembled InAs/GaAs quantum dots by selection of growth steps', J. Appl. Phys., vol. 108, no. 6, p. 63524, Sep. 2010.

[22] D. T. J. Hurle, 'A comprehensive thermodynamic analysis of native point defect and dopant solubilities in gallium arsenide', J. Appl. Phys., vol. 85, no. 10, pp. 6957-7022, May 1999.

[23] D. T. J. Hurle, 'A thermodynamic analysis of native point defect and dopant solubilities in zincblende III-V semiconductors', J. Appl. Phys., vol. 107, no. 12, p. 121301, Jun. 2010. 